\title{
A Note on the Drazin Indices of Square Matrices
}

\author{
Lijun Yu, ${ }^{1}$ Tianyi Bu, ${ }^{1}$ and Jiang $\mathrm{Zhou}^{2,3}$ \\ ${ }^{1}$ College of Automation, Harbin Engineering University, Harbin 150001, China \\ ${ }^{2}$ College of Science, Harbin Engineering University, Harbin 150001, China \\ ${ }^{3}$ College of Computer Science and Technology, Harbin Engineering University, Harbin 150001, China
}

Correspondence should be addressed to Jiang Zhou; zhoujiang04113112@163.com

Received 17 December 2013; Accepted 6 January 2014; Published 10 February 2014

Academic Editors: R. Alperin, V. Nitica, and M. Ronco

Copyright (C) 2014 Lijun Yu et al. This is an open access article distributed under the Creative Commons Attribution License, which permits unrestricted use, distribution, and reproduction in any medium, provided the original work is properly cited.

For a square matrix $A$, the smallest nonnegative integer $k$ such that $\operatorname{rank}\left(A^{k}\right)=\operatorname{rank}\left(A^{k+1}\right)$ is called the Drazin index of $A$. In this paper, we give some results on the Drazin indices of sum and product of square matrices.

\section{Introduction}

For $A \in \mathbb{C}^{n \times n}$, the smallest nonnegative integer $k$ such that $\operatorname{rank}\left(A^{k}\right)=\operatorname{rank}\left(A^{k+1}\right)$ is called the Drazin index of $A$, denoted by $\operatorname{ind}(A)$. The Drazin inverse of $A$ is the unique matrix $A^{D}$ satisfying $A^{k+1} A^{D}=A^{k}, A^{D} A A^{D}=A^{D}$, and $A A^{D}$ $=A^{D} A$, where $k=\operatorname{ind}(A)($ see $[1-3])$. When ind $(A) \leqslant 1, A^{D}$ is called the group inverse of $A$, denoted by $A^{\#}$. If ind $(A)=0$, then $A^{D}=A^{\#}=A^{-1}$. The theory of generalized inverses is an active research field in computational mathematics, and the Drazin index plays an important role in the study of the Drazin (group) inverse. Some results on the Drazin indices of matrices (operators) can be found in [4-9].

For any $A \in \mathbb{C}^{n \times n}$, it is known [10] that there exist nonsingular matrices $P, \Delta$ and nilpotent matrix $N$ such that $A=P\left(\begin{array}{cc}\Delta & 0 \\ 0 & N\end{array}\right) P^{-1}$. In this case, $A^{D}=P\left(\begin{array}{cc}\Delta^{-1} & 0 \\ 0 & 0\end{array}\right) P^{-1}$, and $\operatorname{ind}(A)$ is the smallest nonnegative integer $k$ such that $N^{k}=$ 0 ; that is, $\operatorname{ind}(A)=\operatorname{ind}(N)$. Hence, $\operatorname{ind}(A)$ is the smallest nonnegative integer $k$ such that the group inverse of $A^{k}$ exists.

In this paper, we give some results on the Drazin indices of sum and product of square matrices.

\section{Some Lemmas}

In order to prove our main results, we give some lemmas as follows.
Lemma 1 (see [7]). For any $A \in \mathbb{C}^{n \times n}$ and nonnegative integer $p$, the limit $\lim _{\mathcal{E} \rightarrow 0^{+}} \mathcal{E}^{p}(A+\varepsilon I)^{-1}$ exists if and only if $p \geqslant$ ind $(A)$.

Lemma 2 (see $[8,11])$. Let $M=\left(\begin{array}{cc}A & B \\ 0 & C\end{array}\right)$ be a square complex matrix, where $A$ is square. Then,

$$
\max \{\text { ind }(A) \text {, ind }(C)\} \leqslant \text { ind }(M) \leqslant \text { ind }(A)+\text { ind }(C) \text {. }
$$

Lemma 3 (see $[8,11])$. Let $M=\left(\begin{array}{ll}A & 0 \\ B & C\end{array}\right)$ be a square complex matrix, where $A$ is square. Then,

$$
\max \{\text { ind }(A) \text {, ind }(C)\} \leqslant \text { ind }(M) \leqslant \text { ind }(A)+\text { ind }(C) \text {. }
$$

Lemma 4 (see [12]). Let $M=\left(\begin{array}{cc}A & B \\ 0 & 0\end{array}\right)$ be a square complex matrix, where $A$ is square. Then, $M^{\#}$ exists if and only if $A^{\#}$ exists and $\operatorname{rank}(M)=\operatorname{rank}(A)$.

Lemma 5 (see [12]). Let $M=\left(\begin{array}{ll}A & 0 \\ B & C\end{array}\right)$ be a square complex matrix, where $A$ is square. Then, $M^{\#}$ exists if and only if $A^{\#}, C^{\#}$ exist and $\operatorname{rank}(M)=\operatorname{rank}(A)+\operatorname{rank}(C)$.

Lemma 6 (see [9]). For any $A \in \mathbb{C}^{m \times n}, B \in \mathbb{C}^{n \times m}, \mid \operatorname{ind}(A B)-$ $\operatorname{ind}(B A) \mid \leqslant 1$.

\section{Main Results}

For a square matrix $A$, let $A^{\pi}=I-A A^{D}$. We first give an upper bound for the Drazin index of the sum of two square matrices. 
Theorem 7. Let $P, Q$ be square complex matrices such that $P^{D} Q=0$ and $P Q P^{\pi}=0$. Then,

$$
\text { ind }(P+Q) \leqslant \text { ind }(P)+\text { ind }(Q)-1 \text {. }
$$

Proof. There exist nonsingular matrices $U, \Delta$ and nilpotent matrix $N$ such that $P=U\left(\begin{array}{ll}\Delta & 0 \\ 0 & N\end{array}\right) U^{-1}$. Then, $P^{D}=$ $U\left(\begin{array}{cc}\Delta^{-1} & 0 \\ 0 & 0\end{array}\right) U^{-1}$ and $\operatorname{ind}(P)=\operatorname{ind}(N)$. Suppose that $Q=$ $U\left(\begin{array}{ll}Q_{1} & Q_{2} \\ Q_{3} & Q_{4}\end{array}\right) U^{-1}$, where $Q_{1}$ is a square matrix with the same order as $\Delta$. By $P^{D} Q=0$ we get $Q_{1}=0, Q_{2}=0$. Then, $Q=U\left(\begin{array}{cc}0 & 0 \\ Q_{3} & Q_{4}\end{array}\right) U^{-1}$. By $P Q P^{\pi}=0$ we get $N Q_{4}=0$.

Note that $\operatorname{ind}(P+Q)$ is the smallest nonnegative integer $k$ such that the group inverse of $(P+Q)^{k}$ exists. Since $P+Q=$ $U\left(\begin{array}{cc}\stackrel{\Delta}{Q_{3}} & 0 \\ & \\ Q_{4}\end{array}\right) U^{-1}$, by Lemma 5 , we have ind $(P+Q)=\operatorname{ind}(N+$ $\left.Q_{4}\right)$. Since $N Q_{4}=0$, we have $\varepsilon\left(N+Q_{4}+\varepsilon I\right)=(N+\varepsilon I)\left(Q_{4}+\varepsilon I\right)$. Let $m=\operatorname{ind}\left(Q_{4}\right)+\operatorname{ind}(N)-1$. By Lemma 1 , the limit

$$
\lim _{\varepsilon \rightarrow 0^{+}} \varepsilon^{m}\left(N+Q_{4}+\varepsilon I\right)^{-1}=\lim _{\varepsilon \rightarrow 0^{+}} \varepsilon^{m+1}\left(Q_{4}+\varepsilon I\right)^{-1}(N+\varepsilon I)^{-1}
$$

exists. So ind $(P+Q)=\operatorname{ind}\left(N+Q_{4}\right) \leqslant m=\operatorname{ind}\left(Q_{4}\right)+\operatorname{ind}(N)-$ 1. By Lemma 3, we get ind $\left(Q_{4}\right) \leqslant \operatorname{ind}(Q)$. Since ind $(P)=$ ind $(N)$, we have $\operatorname{ind}(P+Q) \leqslant \operatorname{ind}(P)+\operatorname{ind}(Q)-1$.

Now we give an example to show that the upper bound in Theorem 7 can be attained.

Example 8. Let $P=\left(\begin{array}{cccc}1 & -1 & 1 & 0 \\ 0 & 0 & 0 & 2 \\ 0 & 0 & 0 & 1 \\ 0 & 0 & 0 & 0\end{array}\right), Q=\left(\begin{array}{cccc}1 & -1 & 2 & -2 \\ 1 & -1 & 2 & -2 \\ 1 & -1 & 1 & -1 \\ 1 & -1 & 1 & -1\end{array}\right)$. Then, $\operatorname{ind}(P)=\operatorname{ind}(Q)=2$ and $P^{D}=\left(\begin{array}{cccc}1 & -1 & 1 & -1 \\ 0 & 0 & 0 & 0 \\ 0 & 0 & 0 & 0 \\ 0 & 0 & 0 & 0\end{array}\right)$. By computation, we have $P^{D} Q=0$ and $P Q P^{\pi}=0$. The Drazin index of $P+Q=\left(\begin{array}{cccc}2 & -2 & 3 & -2 \\ 1 & -1 & 2 & 0 \\ 1 & -1 & 1 & 0 \\ 1 & -1 & 1 & -1\end{array}\right)$ is 3. So $\operatorname{ind}(P+Q)=\operatorname{ind}(P)+\operatorname{ind}(Q)-1$.

We can obtain the following result from Theorem 7 .

Corollary 9. Let $P, Q$ be square complex matrices such that $P Q=0$. Then,

$$
\text { ind }(P+Q) \leqslant \text { ind }(P)+\text { ind }(Q)-1 \text {. }
$$

It is known that ind $\left(\begin{array}{ll}A & B \\ C & D\end{array}\right) \leqslant \operatorname{ind}(A)+\operatorname{ind}(D)+2$ under the conditions $C A=0, C B=0$ or $B C=0, D C=0$ (see [13]). A better upper bound is given as follows.

Theorem 10. Let $M=\left(\begin{array}{ll}A & B \\ C & D\end{array}\right)$ be a square complex matrix, where $A$ is square. Then, ind $(M) \leqslant \operatorname{ind}(A)+\operatorname{ind}(D)+1$ if one of the following holds:

(1) $B C=0, D C=0$;

(2) $B C=0, B D=0$;

(3) $C A=0, C B=0$;

(4) $A B=0, C B=0$.

Proof. We only prove part (1). Parts (2)-(4) can be obtained in the same way.
Let $P=\left(\begin{array}{cc}A & B \\ 0 & D\end{array}\right), Q=\left(\begin{array}{cc}0 & 0 \\ C & 0\end{array}\right)$; then, $M=P+Q$. By $B C=0$, $D C=0$, we get $P Q=0$. By Corollary 9 , we have

$$
\text { ind }(M) \leqslant \text { ind }(P)+\text { ind }(Q)-1 \leqslant \text { ind }(P)+1 \text {. }
$$

By Lemma 2, we have

$$
\text { ind }(M) \leqslant \text { ind }(A)+\operatorname{ind}(D)+1 \text {. }
$$

Theorem 11. For $A, B \in \mathbb{C}^{n \times n}$, if $s \geqslant \max \{\operatorname{ind}(A B)$, ind $(B A)\}$, then $(A B)^{s}$ and $(B A)^{s}$ are similar.

Proof. Clearly $(A B)^{0}$ and $(B A)^{0}$ are similar. So we only consider the case $s \geqslant 1$. There exist nonsingular matrices $P, Q$ such that $A=P\left(\begin{array}{cc}I_{r} & 0 \\ 0 & 0\end{array}\right) Q$, where $I_{r}$ is the identity matrix of order $r=\operatorname{rank}(A)$. Suppose that $B=Q^{-1}\left(\begin{array}{ll}B_{1} & B_{2} \\ B_{3} & B_{4}\end{array}\right) P^{-1}$, where $B_{1} \in \mathbb{C}^{r \times r}$. Then,

$$
\begin{array}{ll}
A B=P\left(\begin{array}{cc}
B_{1} & B_{2} \\
0 & 0
\end{array}\right) P^{-1}, & (A B)^{s}=P\left(\begin{array}{cc}
B_{1}^{s} & B_{1}^{s-1} B_{2} \\
0 & 0
\end{array}\right) P^{-1}, \\
B A=Q^{-1}\left(\begin{array}{cc}
B_{1} & 0 \\
B_{3} & 0
\end{array}\right) Q, \quad(B A)^{s}=Q^{-1}\left(\begin{array}{cc}
B_{1}^{s} & 0 \\
B_{3} B_{1}^{s-1} & 0
\end{array}\right) Q .
\end{array}
$$

Since $s \geqslant \max \{\operatorname{ind}(A B)$, ind $(B A)\}$, the group inverses of $(A B)^{s}$ and $(B A)^{s}$ both exist. Lemmas 4 and 5 imply that there exist matrices $X, Y$ such that $B_{1}^{s} X=B_{1}^{s-1} B_{2}$ and $Y B_{1}^{s}=$ $B_{3} B_{1}^{s-1}$. Then,

$$
\begin{aligned}
(A B)^{s} & =P\left(\begin{array}{cc}
B_{1}^{s} & B_{1}^{s} X \\
0 & 0
\end{array}\right) P^{-1} \\
& =P\left(\begin{array}{cc}
I & -X \\
0 & I
\end{array}\right)\left(\begin{array}{cc}
B_{1}^{s} & 0 \\
0 & 0
\end{array}\right)\left(\begin{array}{cc}
I & X \\
0 & I
\end{array}\right) P^{-1}, \\
(B A)^{s} & =Q^{-1}\left(\begin{array}{cc}
B_{1}^{s} & 0 \\
Y B_{1}^{s} & 0
\end{array}\right) Q \\
& =Q^{-1}\left(\begin{array}{cc}
I & 0 \\
Y & I
\end{array}\right)\left(\begin{array}{cc}
B_{1}^{s} & 0 \\
0 & 0
\end{array}\right)\left(\begin{array}{cc}
I & 0 \\
-Y & I
\end{array}\right) Q .
\end{aligned}
$$

Hence, $(A B)^{s}$ and $(B A)^{s}$ are similar.

Corollary 12. For $A, B \in \mathbb{C}^{n \times n}$, if ind $(A B)=s$, then $(A B)^{l}$ and $(B A)^{l}$ are similar for any $l \geqslant s+1$.

Proof. By Lemma 6 and Theorem 11, $(A B)^{l}$ and $(B A)^{l}$ are similar for any $l \geqslant s+1$.

The following corollary is a special case of Theorem 11.

Corollary 13. For $A, B \in \mathbb{C}^{n \times n}$, if $(A B)^{\#}$ and $(B A)^{\#}$ both exist, then $A B$ and $B A$ are similar. 


\section{Conflict of Interests}

The authors declare that there is no conflict of interests regarding the publication of this paper.

\section{Acknowledgments}

This work is supported by the National Natural Science Foundation of China under Grant no. 11371109 and the Fundamental Research Funds for the Central Universities: HEUCF041417.

\section{References}

[1] C. Bu, L. Sun, J. Zhou, and Y. Wei, "Some results on the Drazin inverse of anti-triangular matrices," Linear and Mutilinear Algebra, vol. 61, pp. 1568-1576, 2013.

[2] C. Bu and C. Zhang, "A note on the formulas for the Drazin inverse of the sum of two matrices," Linear Algebra and Its Applications, vol. 439, pp. 565-576, 2013.

[3] C. Deng and Y. Wei, "Representations for the Drazin inverse of $2 \times 2$ block-operator matrix with singular Schur complement," Linear Algebra and Its Applications, vol. 435, no. 11, pp. 27662783, 2011.

[4] R. Bru, J. J. Climent, and M. Neumann, "On the index of block upper triangular matrices," SIAM Journal on Matrix Analysis and Applications, vol. 16, pp. 436-447, 1995.

[5] M. Catral, D. D. Olesky, and P. van den Driessche, "Block representations of the Drazin inverse of a bipartite matrix," Electronic Journal of Linear Algebra, vol. 18, pp. 98-107, 2009.

[6] M. Catral, D. D. Olesky, and P. van den Driessche, "Block representations of the Drazin inverse of a bipartite matrix," Electronic Journal of Linear Algebra, vol. 18, pp. 98-107, 2009.

[7] C. D. Meyer Jr., "Limits and the index of a square matrix," SIAM Journal on Applied Mathematics, vol. 26, no. 3, pp. 469-478, 1974.

[8] C. D. Meyer and N. J. Rose, "The index and the Drazin inverse of block triangular matrices," SIAM Journal on Matrix Analysis and Applications, vol. 33, pp. 1-7, 1977.

[9] Q. Xu, Y. Wei, and C. Song, "Explicit characterization of the Drazin index," Linear Algebra and Its Applications, vol. 436, no. 7, pp. 2273-2298, 2012.

[10] A. Ben-Israel and T. N. E. Greville, Generalized Inverses: Theory and Applications, Springer, New York, NY, USA, 2nd edition, 2003.

[11] R. E. Hartwig and J. M. Shoaf, "Group inverses and Drazin inverses of bidiagonal and triangular Toeplitz matrices," Journal of the Australian Mathematical Society A, vol. 24, pp. 10-34, 1977.

[12] C. Cao, "Some results of group inverses for partitioned matrices over skew fields," Journal of Natural Science of Heilongjiang University, vol. 18, no. 3, pp. 5-7, 2001.

[13] D. S. Cvetković-Ilić, J. Chen, and Z. Xu, "Explicit representations of the Drazin inverse of block matrix and modified matrix," Linear and Multilinear Algebra, vol. 57, no. 4, pp. 355364, 2009. 


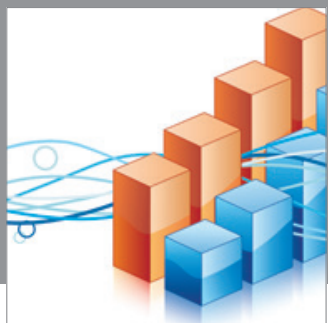

Advances in

Operations Research

mansans

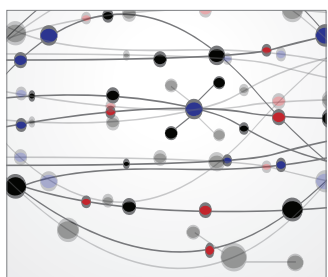

The Scientific World Journal
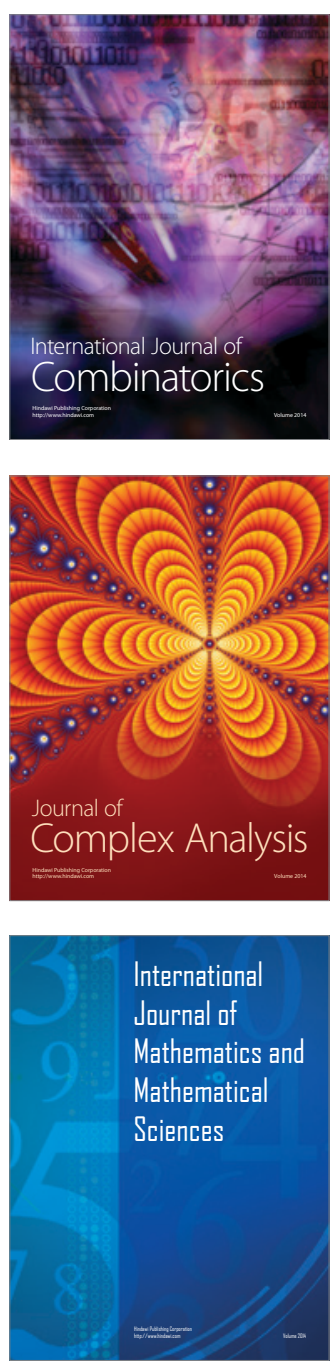
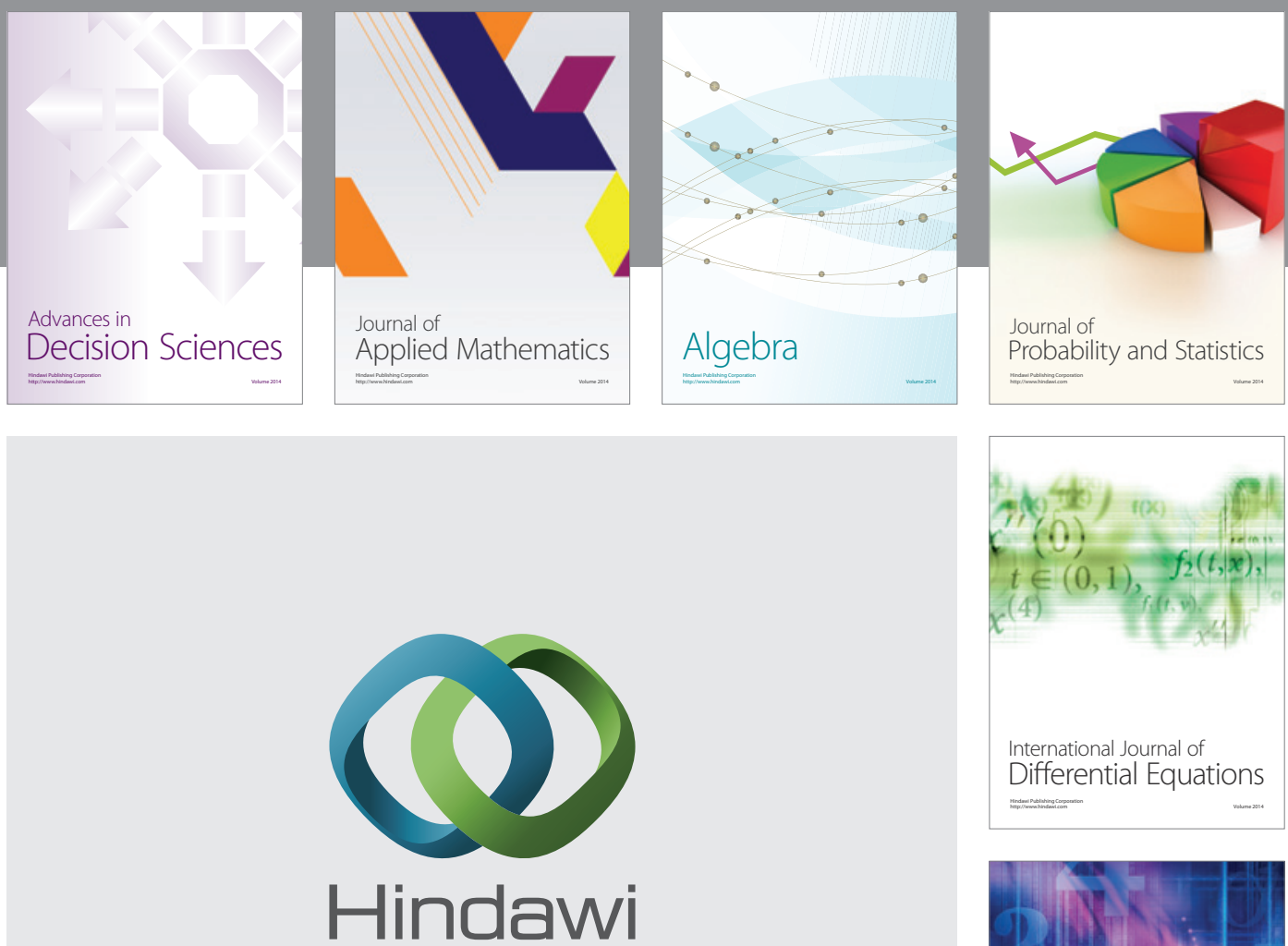

Submit your manuscripts at http://www.hindawi.com
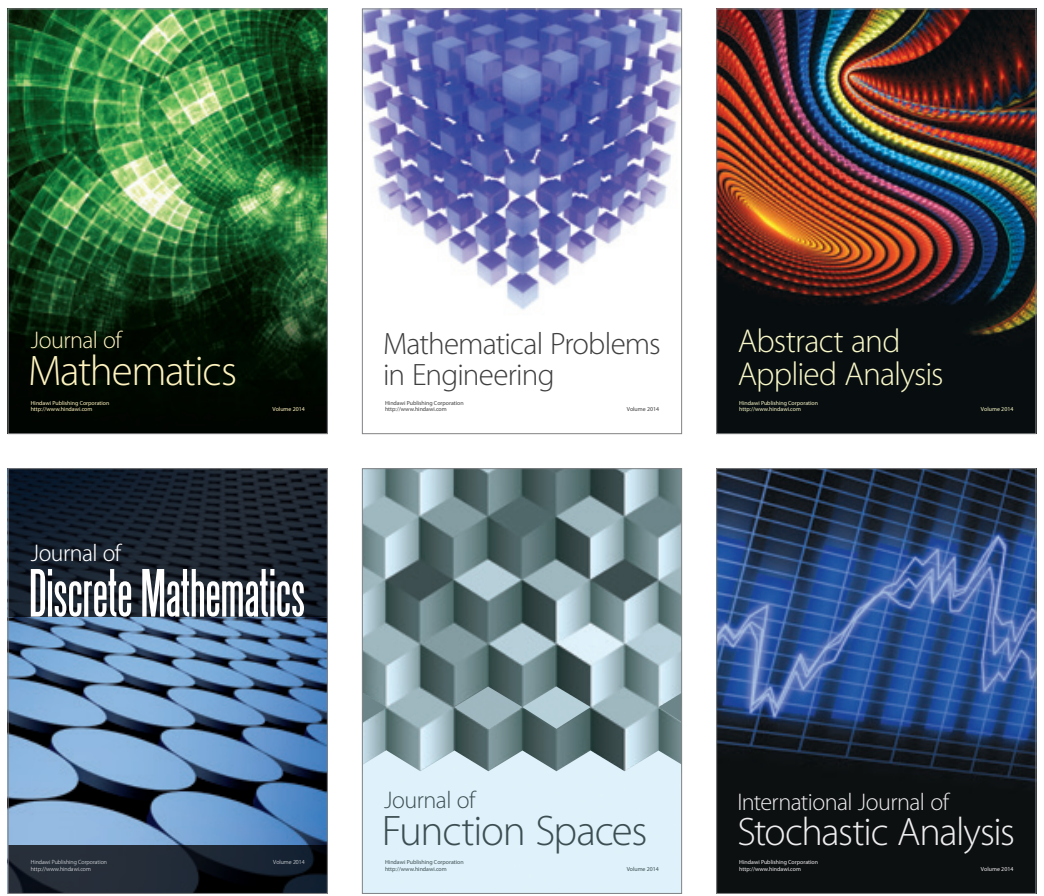

Journal of

Function Spaces

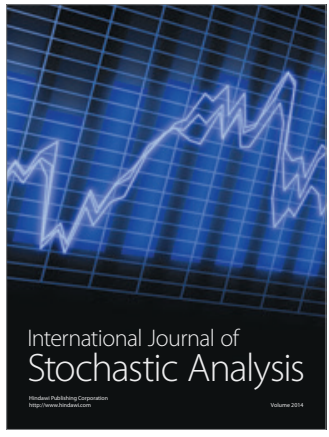

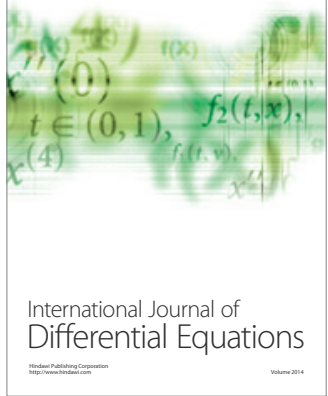
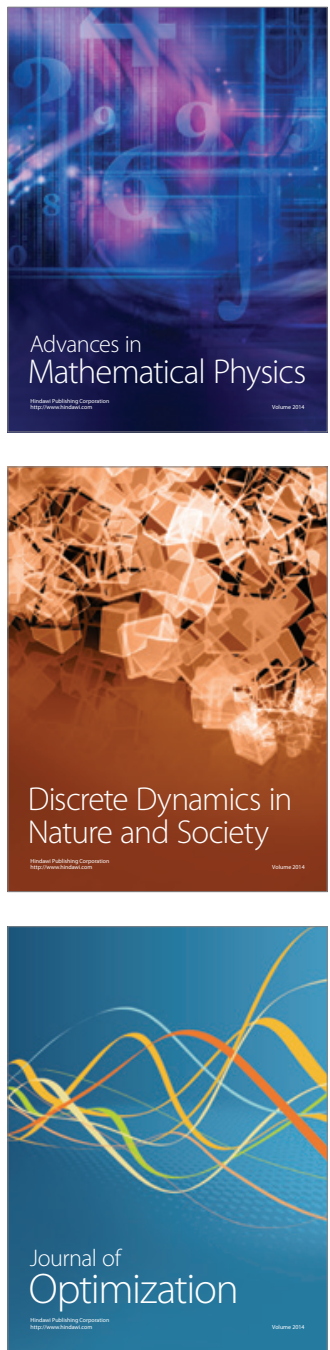